\title{
External ocular findings in lupus erythematosus: a clinical and immunopathological study
}

\author{
Peggy Frith, S M Burge, P R Millard, F Wojnarowska
}

\begin{abstract}
A selected group of 18 patients with systemic lupus erythematosus (SLE) and 30 patients with chronic cutaneous lupus erythematosus (CCLE) showed an unexpectedly high incidence of problems involving the globe or eyelids. Five SLE patients had recurrent episcleritis, two CCLE patients had lower tarsal plaques, and two further CCLE patients had erosion of the lower lid margins associated with conjunctival scarring and symblepharon. This association has not previously been reported. There was an unexpectedly high incidence of deposition of immunoreactants in a linear pattern at the basement membrane zone in normal bulbar conjunctiva, which occurred in both SLE (42\%) and CCLE (50\%). The significance of these findings is discussed. We believe surface ocular problems in lupus erythematosus to be under-reported and that direct immunofluorescence of bulbar conjunctival biopsy might be helpful in diagnosis.
\end{abstract}

Lupus erythematosus (LE) is diagnosed and defined according to clinical and laboratory criteria. Patients with systemic LE (SLE) have manifestations outside the skin, and a high frequency of circulating antinuclear antibodies.' Patients with chronic cutaneous LE (CCLE) have scarring disease confined to the skin and rarely have circulating antinuclear antibodies.

Ulceration of the mouth is quite common in SLE, occurring in up to $45 \%$ of patients, ${ }^{23}$ and is accepted as one of the clinical diagnostic criteria. Chronic oral plaques may also develop in up to $20 \%$ of patients with CCLE, ${ }^{2+}$ but in most the presence of characteristic skin lesions is a guide to the aetiology. In both conditions the oral lesions have similar histopathological abnormalities to those found in skin.

Direct immunofluorescence (IMF) of skin shows deposition of immunoreactants at the basement membrane zone in some patients with SLE - up to $80 \%$ in uninvolved skin ${ }^{5}$ - and in CCLE in biopsies from skin or lip lesions. ${ }^{67}$ The significance of this finding is uncertain in terms of prognosis and pathogenesis, but it may be helpful in confirming the diagnosis if other confounding disorders such as pemphigoid are

In patients with SLE reports of surface ocular abnormalities are confined to passing references to a low incidence of conjunctivitis, episcleritis, or scleritis, and assessment of the incidence of sicca. ${ }^{8-11}$ In patients with CCLE Kaposi mentions involvement of the eyelid in his description of discoid LE in 1883. ${ }^{12}$ Subsequent

reports are sparse, describing focal tarsal con- junctival lesions, ${ }^{13-15}$ or involvement of the lid margin, ${ }^{131+16-20}$ reviewed by Huey et al. ${ }^{18}$

Our group has previously made a comparison of clinical and immunofluorescent findings in the skin, mouth, and conjunctiva in cicatricial and bullous pemphigoid. ${ }^{21}$ In these patients, presenting with skin or mouth lesions, we found an unexpectedly high rate of asymptomatic ocular abnormalities, including positive conjunctival IMF. We were therefore interested to study a group of patients with SLE and CCLE in a similar way to see if ocular features were present even if ocular symptoms were absent.

\section{Patients and methods}

Patients were recruited from the dermatology, rheumatology, and general medical clinics. Although they were not known to have ocular problems at presentation, there was a referral bias in favour of patients with ocular symptoms or signs. From a pool of 53 SLE and 68 CCLE patients assessed for a study of all mucosal sites 18 patients with SLE and 30 with CCLE were referred.

Systemic LE. Eighteen patients were considered to have SLE by American Rheumatism Association 1982 criteria; 16 were female and two male. All except one had skin and joint involvement, and eight had renal disease. Their ages ranged from 27 to 69 years (mean $46 \cdot 5$ years). The disease duration ranged from 9 months to 31 years (average 11.6 years). Sixteen patients were receiving immunosuppressive treatment with oral prednisolone $(2 \cdot 5-30 \mathrm{mg} /$ day, average $9 \mathrm{mg}$ ), some also with azathioprine and/or antimalarials. One patient with recent presentation of an urticarial LE rash was untreated, as were two patients with mild or inactive disease.

Chronic cutaneous $L E$. Thirty patients fulfilled clinical and histopathological criteria for CCLE, and in 18 this was confined to the head and neck. Histological criteria were the presence of follicular plugging, lichenoid lymphocytic infiltrate, and basal cell liquefaction. Twenty patients were female, 10 male. Their ages ranged from 22 to 77 years (mean 47.5 years). The disease duration ranged from 2 to 54 years (mean 12.8 years). Most were controlled with topical corticosteroid and sunscreen preparations; five patients were also taking antimalarials.

IMF control patients. Bulbar conjunctival biopsies were obtained from 50 patients at the time of surgery for unrelated conditions such as cataract, squint, and retinal detachment. No patient had known primary conjunctival disease. Twenty six were female, 24 male, and their ages ranged from 1 to 88 years (mean 63 years).

Clinical methods. A detailed history of ocular excluded.

\section{Oxford} \\ Correspondence to: Dr P A \\ Frith, Eye Hospital, Walto \\ Accepted for publication \\ 7 September 1989 \\ Department of \\ Histopathology, Jo \\ Radcliffe
}


TABLE I Clinical ocular signs in SLE and CCLE

\begin{tabular}{|c|c|c|}
\hline & Systemic $L E$ & $\begin{array}{l}\text { Chronic cutaneous } \\
\text { LE }\end{array}$ \\
\hline No. of patients & $18(\%)$ & $30(\%)$ \\
\hline $\begin{array}{l}\text { Lids } \\
\text { Blepharitis } \\
\text { Lid plaque } \\
\text { Lid scarring } \\
\text { Symblepharon }\end{array}$ & $\begin{array}{l}3(17) \\
0 \\
0 \\
0\end{array}$ & $\begin{array}{l}5(17) \\
2(7) \\
2(7) \\
2(7)\end{array}$ \\
\hline $\begin{array}{l}\text { Globe } \\
\text { Episcleritis, recurrent } \\
\text { Episcleral injection, chronic } \\
\text { Dryness }\end{array}$ & $\begin{array}{l}5(28) \\
0 \\
2(11)\end{array}$ & $\begin{array}{l}0 \\
2(7) \\
2(7)\end{array}$ \\
\hline
\end{tabular}

symptoms was taken, particularly of redness of the lids or globe, soreness, discomfort, or dryness, and itching or discharge. These were graded subjectively as mild, moderate, or severe. The lid surfaces and margins and the everted conjunctival surface and globe were examined by the slit-lamp by the same ophthalmologist (PF). Schirmer's testing with standard strips for 5 minutes was recorded on a single occasion in each patient.

Biopsies. Biopsies were taken from consenting patients at a standard site from the upper bulbar conjunctiva, which was clinically normal and not sun-exposed. The simple technique with topical anaesthesia has been previously described. ${ }^{22}$ Tissue snips $2 \mathrm{~mm}$ long were embedded in optimal cutting temperature (OCT) medium and snap frozen. The conjunctiva of 12 SLE and 21 CCLE patients was biopsied (that is, $2 / 3$ in both groups). All patients except three also had biopsies of normal forearm skin and lip to compare the incidence of positive IMF at the three sites.

Cryostat sections were examined by direct immunofluorescence using fluorescein isothiocyanate (conjugated) (FITC) labelled antihuman IgG, A, M, C3, C4, and Clq (Hoechst UK Ltd). The assessing pathologist (PRM) was unaware of the patient's status.

The study was carried out with the informed consent of the patients and the approval of the Ethical Committee.

\section{Results}

The clinical findings in both groups are summarised in Table I.

SLE

Twelve patients, or two-thirds, reported ocular symptoms. Intermittent redness of one or both

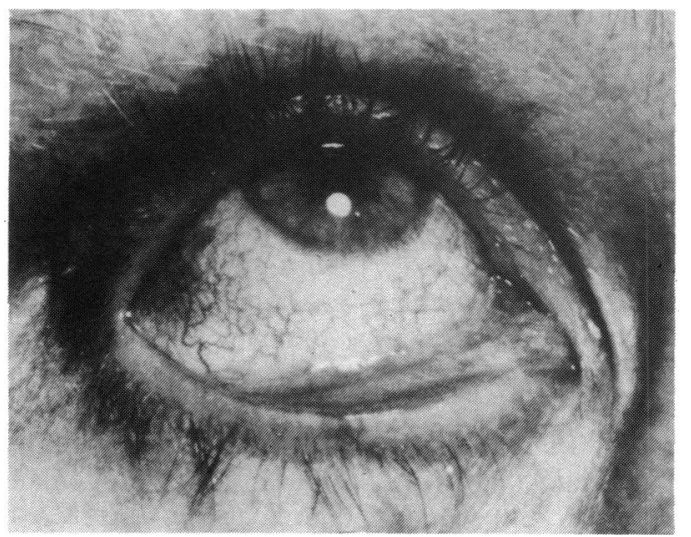

Figure 1: SLE patient showing episcleritis.

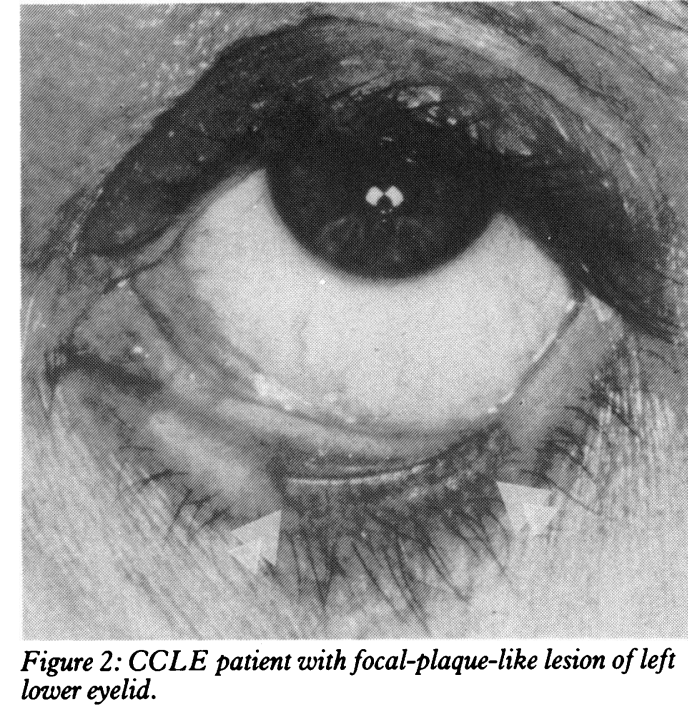

globes with moderate discomfort and no discharge was suggestive of recurrent episcleritis in five patients $(28 \%)$. This was confirmed in each case by observation during an attack (Fig 1). One patient mentioned recurrent redness of the lids, and three patients had non-specific, non-scarring blepharitis. Three patients described itching, and three others swelling of the lids without an atopic background. Two patients, one of whom was asymptomatic, had significant dryness, with a Schirmer's test of less than $10 \mathrm{~mm}$ in both eyes. Thus overall nearly half the patients had signs which could have been related to the underlying SLE.

\section{CCLE}

Seventeen patients, just over half, reported symptoms. Three reported persistent redness of the globe and 11 redness of the lids. Four mentioned itching; in one with ocular scarring this was intermittently severe.

Ten patients, or one-third, had ocular signs. In five there was non-specific blepharitis without scarring. In contrast two other patients had focal plaque-like lesions of the lower lid. These lesions were circumscribed, red, oedematous areas which involved the lid margin and extended on to the lower tarsal conjunctival surface. The lesions were bilateral in one patient, unilateral in the other (Fig 2). They responded well to topical steroid treatment, but in the patient with more extensive bilateral lesions these have recurred as treatment was reduced.

Two patients who both had CCLE of long duration ( 30 and 54 years) had striking lower lid involvement with pronounced scarring and tissue loss, resembling their destructive facial skin lesions. One of these patients said that her eyelids were intermittently very itchy; the other had only minor discomfort. In both patients the lids were thickened and red, with focal loss of lid margin architecture and tissue, including lash loss. There was a mild degree of ectropion in each case, but the lower puncta were open. The mucocutaneous junction was disorganised and keratinised. In addition, in both patients eversion of the lower lid showed the lower tarsal conjunctiva to be scarred, with shrinkage of the 


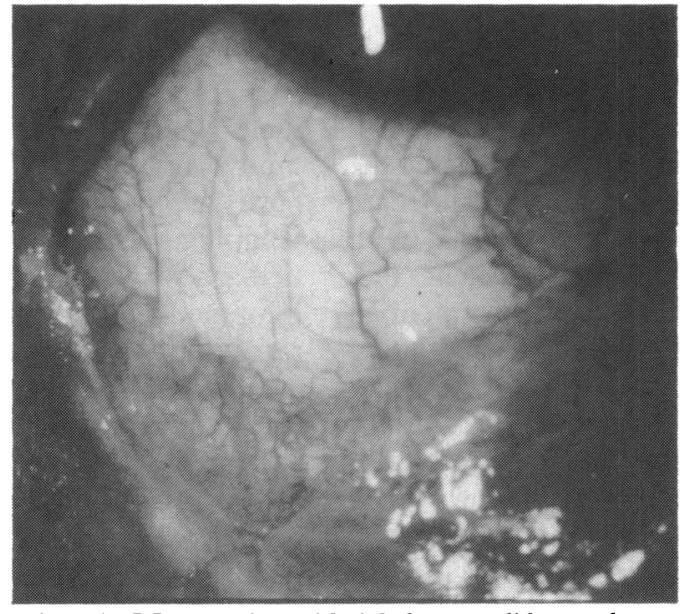

Figure 3: CCLE patient with right lower eyelid everted, showing scarring of lid margin and lower conjunctival formix.

lower fornix and symblepharon formation (Fig 3). The medial canthal architecture was also lost. The upper lids were normal. The bulbar conjunctival and episcleral vessels were persistently dilated. One patient had central stromal corneal scarring with reduced vision which he attributed to trauma in childhood, but otherwise the corneae were normal. Two patients gave reduced results in Schirmer's test.

CONJUNCTIVAL IMMUNOFLUORESCENCE Twelve, that is two-thirds, of the SLE patients had conjunctival biopsy. Five biopsies showed a linear pattern of immunoreactants deposited at the basement membrane zone $(42 \%)$. In four cases this was IgG alone (Fig 4), in the other Clq alone.

Twenty-one, also two-thirds, of the patients with CCLE were biopsied and $10(48 \%)$ showed an identical linear pattern in the conjunctiva. All had IgG, and one, a patient who had symblepharon, also had linear IgA and C3.

In neither group was there correlation between clinical and IMF findings, many IMF positive patients having no significant symptoms or signs. Of SLE patients with episcleritis two did and two did not have immune deposits, and one was not biopsied. Both CCLE patients with scarring had positive linear IMF.

A comparison of positive IMF in skin and in lip mucosa in the same patients (Tables II and III) showed that in both SLE and CCLE the rate of IMF positivity was highest in conjunctiva

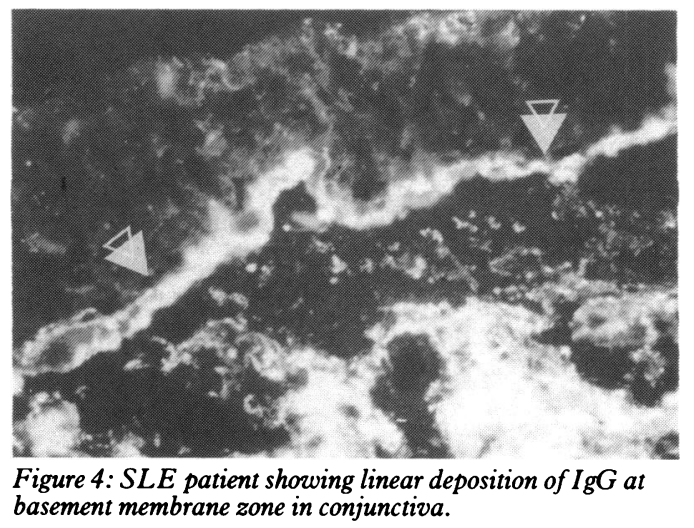

TABLE II Systemic LE: tissue immunofluorescence at three uninvolved sites

\begin{tabular}{llll}
\hline & Conjunctiva & Skin & Lip mucosa \\
\hline Total no. biopsies & 12 & 16 & 16 \\
No. positive band & 5 & 5 & 4 \\
\% Positive band & 42 & 31 & 25 \\
\hline
\end{tabular}

TABLE III Chronic cutaneous LE: tissue immunofluorescence at three uninvolved sites

\begin{tabular}{llll}
\hline & Conjunctiva & Skin & Lip mucosa \\
\hline Total no. biopsies & 21 & 30 & 29 \\
No. positive band & 10 & 1 & 1 \\
\% Positive band & 48 & 3 & 3 \\
\hline
\end{tabular}

compared with skin and lip, particularly in CCLE.

In control patients a positive linear result was found in five of 50 conjunctival biopsies. One patient had had a renal transplant and could reasonably be excluded, as the immune deposits might have been related to the underlying condition. Another positive control patient, included in analysis, had thyroid eye disease. Thus in three of 49 control patients $(6 \%)$ linear IgG was present, and in a further patient linear IgM.

\section{Discussion}

This study of the prevalence of clinical and immunopathological abnormalities of the ocular surface in LE has shown a surprisingly high rate in both systemic and chronic cutaneous LE.

\section{CLINICAL FEATURES}

Previous reports date from 1907. The first report in English $^{13}$ (1932) describes three patients with conjunctival lesions and reviews reports of nine other patients, three with conjunctiva, four with lid, and two with both sites involved. Since then there have been occasional reports totalling eight patients with conjunctival ${ }^{14}$ is and 29 with lid $^{1416-20}$ lesions thought to be related to associated lupus. It is not always possible to be sure if these are cases of SLE or CCLE.

Five patients showed swelling of the lids associated with lupus profundus, and panniculitis was shown on facial skin biopsy. ${ }^{17}$ Otherwise lid lesions were characterised by blepharitis with scaling, madarosis, and non-contractile scarring in some cases. Biopsy of the lid margin in four case $^{1820}$ showed a similar histology to LE skin lesions elsewhere, with follicular plugging, oedema, basal cell liquefaction, and a dense, patchy, cellular infiltrate. The lesions were distinguished clinically from chronic blepharitis by the association with skin lesions of LE in all but three cases, and there was often a history of exacerbation by sunlight. In three case ${ }^{20}$ the lid lesions were the only abnormality but proved to be typical of CCLE on biopsy.

Conjunctival lesions have been described as circumscribed, red, oedematous, velvety areas on the upper or lower tarsal surface, which heal spontaneously or in response to systemic treatment, sometimes leaving atrophic depressed scars. ${ }^{14}$ is

We consider that in our group of SLE patients that recurrent episcleritis in five out of $18(28 \%)$ 
is likely to be related to the underlying LE. One patient presented with red eyes as her first manifestation, progressing within months to joint and urticarial skin involvement with positive antinuclear antibodies and characteristic skin histopathology. Her eyes tended to flare with her systemic disease, but subsided with increase in systemic and topical treatment. It is reasonable to consider this a manifestation of an immune complex disorder, though circulating complexes were not measured in our patients. We cannot prove an association, but a rate of $28 \%$ seems higher than expected by chance even with a referral bias in favour of ocular problems. In this small group we did not find scleritis, which has been occasionally reported in SLE. ${ }^{23}$

In the CCLE group five patients (17\%) had blepharitis with no special features, and this could be an incidental finding. However, a further four patients had lid involvement characteristic of the condition, though lid biopsies were not performed. Two of these patients had circumscribed plaque-like blepharitis of the lower lid margin, extending inwards on to the tarsal surface, with striking redness of the lesions well demarcated from the adjacent normal tissue. These responded rapidly to topical corticosteroid application, leaving no scars, but in one patient the lesions recurred. In one of these patients a single asymptomatic eyelid lesion was associated with marked oral involvement, and a careful search showed small plaques of CCLE behind one ear. The diagnosis of LE was made by positive linear IMF in oral tissue. Thus the lid lesion was helpful in suggesting the diagnosis.

Two further patients with CCLE had ocular findings which have not previously been described and which were strikingly similar to each other. Both patients had very long-standing facial cutaneous disease with photosensitivity, and both showed striking loss of lower lid tissue with scarring. The scarring also involved the lower tarsal conjunctiva, with symblepharon formation. This feature resembled cicatricial pemphigoid (CP), though lid margin involvement has not been reported in CP, and we believe this to be the first report of symblepharon in CCLE.

Clinical features in non-ocular mucosal surfaces in a group of $121 \mathrm{LE}$ patients from which the present study was drawn are being reported separately. ${ }^{24}$ Oral lesions were found in $10 \%$, nasal mucosal lesions in $6 \%$ overall, and vulval lesions in $5 \%$ of the female patients.

TISSUE IMMUNOFLUORESCENCE

There are no previous reports of tissue direct immunofluorescence findings in cases with ocular involvement, except for a single case with biopsy of oedematous lid skin which showed positive linear IgG deposition in a broad granular pattern. ${ }^{19}$ This is the pattern found in up to $60 \%$ of patients with cutaneous LE, known as the 'lupus band'. We did not investigate IMF in eyelid skin.

Conjunctival IMF findings in LE patients have not to our knowledge been previously described. Our findings of IMF in three different tissue sites are summarised (Tables II and III) and have been reported separately. ${ }^{25} \mathrm{We}$ were surprised by the high incidence of conjunctival linear immunodeposits compared with uninvolved skin and lip in the same patients. We have previously found the same pattern in pemphigoid patients. ${ }^{21}$ The comparison is particularly striking in our CCLE group, in which immunodeposits in uninvolved skin and lip were rare but were found in $48 \%$ of uninvolved bulbar conjunctiva. Perhaps circulating antibodies are bound more readily to conjunctiva or are cleared less rapidly from it.

The origin and significance of the immunodeposits at any site is unclear. Elution studies in man $^{26}$ suggest that in skin the antibody has antinDNA specificity, and studies in mice ${ }^{27}$ and in humans $^{6}$ that they are related to circulating nDNA levels. It has been postulated that in-situ complexes form as nDNA is released from degenerating surface keratinocyte nuclei, perhaps particularly in response to solar irradiation, ${ }^{28}$ but our finding of a band in nonkeratinised, non-sun-exposed conjunctiva suggests other mechanisms may be involved.

In oral mucosa, linear IgG in lesional tissue is a good discriminator for LE. In a large series ${ }^{7}$ a positive band was detected in $73 \%$ of CCLE and $100 \%$ of SLE associated lesions, but was absent from lesions of lichen planus or leucoplakia. In our large control group we found a low (6\%) incidence of linear IgG in normal bulbar conjunctiva, suggesting that the association of this pattern with both SLE and CCLE is significant.

We conclude that ocular surface disorders such as episcleritis in SLE and lid and conjunctival lesions in CCLE are not uncommon, and may be under-reported, particularly as symptoms may be minor or absent. The ocular features can occur early, and may be sufficiently characteristic to suggest the diagnosis of LE. Positive linear IMF in uninvolved bulbar conjunctiva is surprisingly common in both SLE and CCLE, and this finding deserves further study, as it may be of value diagnostically. The association of CCLE with symblepharon is a new finding, and raises interesting comparisons with cicatricial pemphigoid and questions about pathogenesis of autoimmune disorders involving the basement membrane zone.

This study was supported by the Arthritis and Rheumatism Council and the Oxford RHA.

The authors would like to thank all the clinicians who referred patients for inclusion in the study in particular Dr J Ledingham, patients for inclusion in the study, in particular Dr J Ledingham, Benjamin for supplying conjunctival tissue from control patients, and the MLSO staff of the John Radcliffe Histopathology and the MLSO staff of the John Radcliffe H
Department for preparing and staining the biopsies.

1 Tan EM, Cohen AS, Fries JF, et al. The 1982 revised criteria for the classification of systemic lupus erythematosus. Arthritis Rheum 1982; 25: 1271-7.

2 Schiodt M. Oral manifestations of lupus erythematosus. Int $f$ Oral Surg 1984; 13: 101-47.

3 Jonsson R, Heyden G, Westberg NG, Nyberg G. Oral mucosal lesions in SLE. A clinical, histopathological and immunopathological study. F R heumatol 1984; 11: 38-42.

4 Andreas JO. Oral manifestations in discoid and systemic lupus erythematosus. Acta Odontol Scand 1964; 22: 295-310.

5 Jordan RE, Schroeter AL, Winkelman RK. Dermalepidermal deposition of complement components and epidermal deposition of complement compone
properdin in SLE. Br 7 Dermatol 1975; 92: 263-71.

properdin in SLE. Br f Dermatol 1975; 92: 263-71.
6 Provost TT. Lupus band test. Int $\mathcal{F}$ Dermatol 1981; 20:475-81.

6 Provost TT. Lupus band test. Int F Dermatol 1981; 20: 475-81. of immunoglobulins, complement and fibrinogen in oral LE, lichen planus, and leukoplakia. Oral Surg 1981; 51:

8 Dubois EI, Tuffanelli DL. Clinical manifestations of SLE: analysis of 520 cases. $\mathcal{F A M A} 1964 ; 190$ : 104-11. 
9 Gold DH, Morris DA, Henkind P. Ocular findings in SLE. Brf Ophthalmol 1972; 56: 800-4.

10 Williamson J. Incidence of eye disease in cases of connective tissue diseases. Trans Ophthalmol Soc UK 1974; 94: 742-52.

11 Ramos-Niembro F, Alarcon-Segovia D Development of sicca symptoms in SLE patients with existing subclinical abnormalities. Arthritis Rheum 1979; 22: 935-6.

12 Kaposi M. Pathologie und Therapie der Hautkrankheiten. Vorlesungen für Praktische Aertze und Studirende. 2nd ed. Vienna: Urban and Scwartzenberg, 1883: 642.

13 Klauder JV, Delong P. LE of conjunctiva, eyelids and lid margins. Arch Ophthalmol 1932; 7: 856-67.

14 Delong P, Klauder JV. LE of eyelids and conjunctiva. Arch Ophthalmol 1936; 16: 321-2.

15 Vilanova von X, Cardenal C, Capdevila JM. Chronischer Lupus erythematodes der Conjunctiva. Dermatologica 1956; 113: $226-31$

16 Nowinski T, Vitaliano B, Naidoff $M$, Parrish R. Ocular involvement in LE profundus (panniculitis). Ophthalmology 1982; 89: 1149-54.

17 Kearns W, Wood W, Marchese A. Chronic cutaneous lupus involving the eyelid. Ann Ophthalmol 1982; 14: 1009-10.

18 Huey C, Jakobiec FA, Iwamoto T, et al. Discoid lupus erythematosus of the eyelids. Ophthalmology 1983; 90: 1389 98 .

19 Donzis PB, Insler MS, Buntin DM, Gately LE. DLE involv- ing the eyelids. Am f Ophthalmol 1984; 98: 32-6.

20 Tosti A Tosti G DLE solely involving the eyelids: report of three cases. 7 Am Acad Dermatol 1987; 16: 1259-60.

21 Venning VA, Frith PA, Bron AJ, Millard P, Wojnarowska F Mucosal involvement in bullous and cicatricial pemphigoid Mucosal involvement in bullous and cicatricial pemphigoid. A clinical and im

22 Frith PA, Venning VA, Wojnarowska F, Millard PR, Bron AJ. Conjunctival involvement in cicatricial and bullous pemphigoid: a clinical and immunopathological study. $\mathrm{Br} \mathcal{F}$ Ophthalmol 1989; 73: 52-6.

23 Watson PG, Hayreh SS. Scleritis and episcleritis. $\mathrm{Br} \mathcal{F}$ Ophthalmol 1976; 60: 163-91.

24 Burge SM, Frith PA, Juniper RP, Wojnarowska F. Mucosa involvement in systemic and chronic cutaneous lupus involvement in systemic and chronic

25 Burge SM, Frith PA, Millard PR, Wojnarowska F. The lupus band in oral mucosa, conjunctiva and skin. Br $\mathcal{F}$ Dermatol. (In press).

26 Landry $M$, Sams WM. SLE: studies of the antibodies bound to skin. F Clin Invest 1973; 52: 1871-80.

27 Gilliam JN. Significance of cutaneous immunoglobulin deposits in LE and NZB/NZW F1 hybrid mice. $\mathcal{f}$ Invest Dermatol 1975; 65: 154-61.

28 Zamansky GB [Editorial]. Sunlight induced pathogenesis in SLE. F Invest Dermatol 1985; 85: 179-80. 